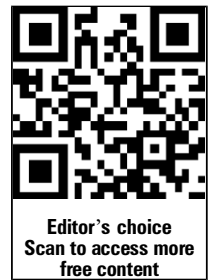

For numbered affiliations see end of article.

\section{Correspondence to} Professor Simon Murch, Division of Metabolic and Vascular Health, Warwick Medical School, Clifford Bridge Road, Coventry CV2 2DX, UK; s.murch@warwick.ac.uk

Received 14 March 2013 Revised 5 June 2013 Accepted 29 June 2013
To cite: Murch $\mathrm{S}$, Jenkins $\mathrm{H}$, Auth $\mathrm{M}$, et al. Arch Dis Child 2013:98:806-811.

\title{
Joint BSPGHAN and Coeliac UK guidelines for the diagnosis and management of coeliac disease in children
}

Simon Murch, ${ }^{1}$ Huw Jenkins, ${ }^{2}$ Marcus Auth, ${ }^{3}$ Ronald Bremner, ${ }^{4}$ Assad Butt, ${ }^{5}$ Stephanie France, ${ }^{6}$ Mark Furman, ${ }^{7}$ Peter Gillett, ${ }^{8}$ Fevronia Kiparissi, ${ }^{9}$ Maureen Lawson, ${ }_{10}^{10}$ Bruce McLain, ${ }^{11}$ Mary-Anne Morris, ${ }^{12}$ Sarah Sleet, ${ }^{13}$ Matthew Thorpe ${ }^{14}$

\section{ABSTRACT}

The revised BSPGHAN guidelines for the diagnosis and management of coeliac disease represent an important shift in diagnostic strategy, aimed at simplifying and shortening the diagnostic process in selected cases. Guidance is given concerning the indications for testing for coeliac disease, which is still significantly underdiagnosed in the UK. While screening data suggest a likely incidence of 1 in 100 persons, only 10\%-20\% of this figure is currently being diagnosed.The BSPGHAN guidelines follow the new ESPGHAN guidelines in overall diagnostic strategy, while providing more didactic stratagems, which should be of assistance for paediatricians in specialties other than gastroenterology.

\section{BSPGHAN GUIDELINE}

This guideline extends the earlier BSPGHAN guideline (based on NASPGHAN Coeliac Guideline of $2005^{1}$ and the original guideline from the Welsh Paediatric Gastroenterology $\mathrm{MCN}^{2}$ ) to incorporate the changed ESPGHAN 2012 diagnostic guideline. ${ }^{3}$ An outline of these guidelines (figures 1 and 2) are also available to download from the BSPGHAN and Coeliac UK websites. The British Society of Gastroenterology (BSG) Coeliac Guideline for Adult Coeliac Disease, which differs in respect of biopsy stratagem, is available on the BSG website http://www.bsg.org.uk.

Coeliac disease (CD) is not simply a gastrointestinal condition but an immune-mediated systemic disorder, strongly dependent on the human leukocyte antigen (HLA)-DQ2 and DQ8 haplotypes. It is elicited by gluten and related prolamines in genetically susceptible individuals and characterised by a variable combination of gluten-dependent clinical manifestations, CD-specific antibodies and enteropathy. ${ }^{3-7}$ Screening studies have shown prevalence much higher than previously recognised, and there is evidence of an increased incidence of both classic and non-classic presentations in UK children. ${ }^{8}$

BSPGHAN recommends that all patients with suspected CD should have their diagnosis established by a paediatric gastroenterologist and their follow-up under the care of a paediatric gastroenterologist or a paediatrician with a special interest in CD, with access to appropriately skilled paediatric dietetic services. ${ }^{9}$
Box 1 Symptomatic children (gastrointestinal tract and non-gastrointestinal tract symptoms)

Persistent diarrhoea

- Faltering growth, idiopathic short stature

- Abdominal pain, vomiting, abdominal distension

- Constipation

- Dermatitis herpetiformis

- Dental enamel defects

- Osteoporosis/pathological fractures

- Delayed menarche

- Unexplained anaemia or iron deficient anaemia unresponsive to treatment

- Recurrent aphthous stomatitis

- Unexplained liver disease

- Lassitude/weakness

\section{Box 2 Asymptomatic but with associated} condition (estimated lifetime prevalence)

Type I diabetes ( $\geq 8 \%$ )

- Selective IgA deficiency $(1.7 \%-7.7 \%)$

- Down (5\%-12\%), Williams (8.2\%) and Turner $(4.1 \%-8.1 \%)$ Syndromes

- Autoimmune thyroiditis ( 15\%)

- Autoimmune liver disease

- Unexplained raised transaminases without known liver disease

- Intussusception

- Dermatitis herpetiformis

- Relatives of coeliac patient:

- First-degree relative $(\sim 10 \%)$

- HLA-matched sibling ( 30\%-40\%)

- Monozygotic twin ( 70\%)

Who to test

The prevalence of CD is estimated to be $1: 100$ in the UK. Universal population screening is not currently advised. However, there should be a low threshold for investigating both symptomatic children and those with associated conditions, as it is recognised that approximately $90 \%$ of cases remain undiagnosed (boxes 1 and 2). 


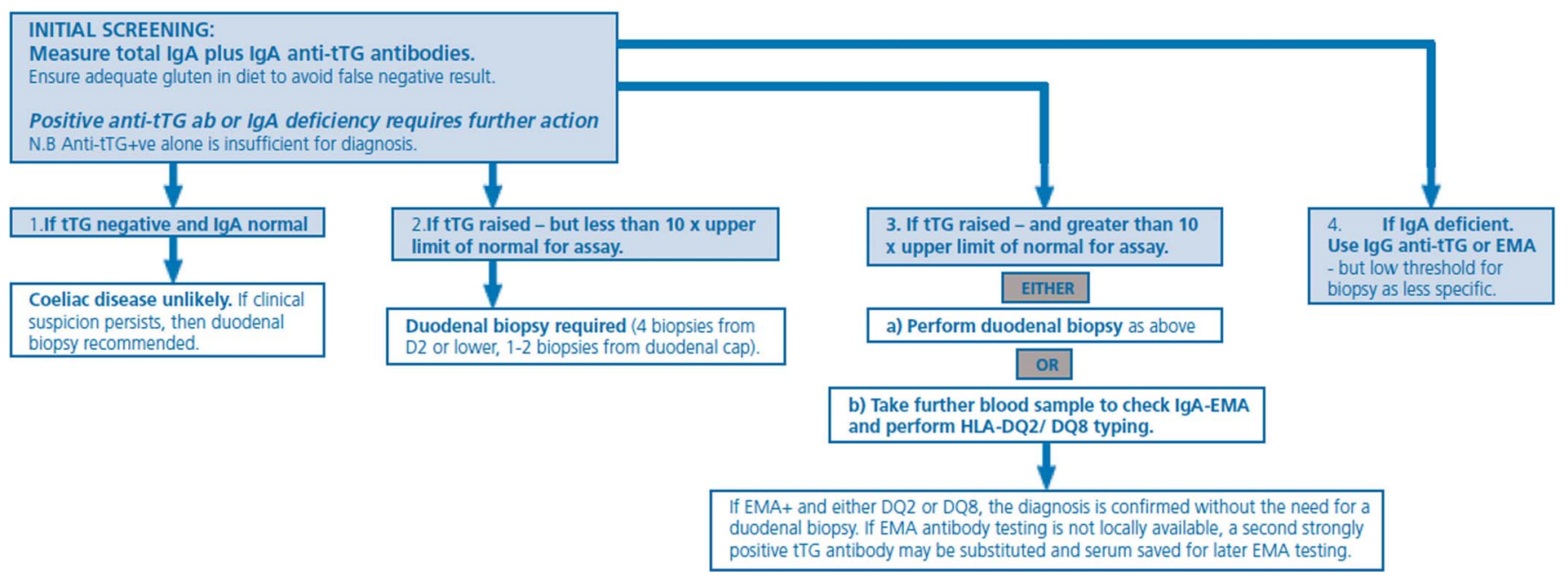

Figure 1 Outline stratagem for symptomatic children.

CD should also be considered in juvenile idiopathic arthritis, epilepsy with associated intracranial calcification and unexplained neurological problems (palsies, neuropathies, migraine).

If screening parents or patients, families should be advised pretesting about relative risks of untreated CD and the need for biopsy and gluten-free diet (GFD) should the blood tests come back positive.

Ensure adequate gluten intake pretesting and consult dietician if formal assessment required (see section 3 and appendix 1). If possible, test when venesection done for other reasons (annual review, etc).

\section{Blood antibody testing to screen for CD}

Initial screening requires estimation of total $\operatorname{IgA}$ and $\operatorname{IgA}$ antitissue transglutaminase (tTG) antibodies. Findings of positive anti-tTG antibody or IgA deficiency require further diagnostic action-usually a small bowel biopsy or (only in certain specific circumstances detailed below) a second blood test for endomysial (EMA) testing and HLA-DQ typing. ${ }^{3}$

In the case of IgA deficiency, IgG anti-tTG or IgG anti-EMA may help to decide on need for biopsy. However, neither IgG anti-tTG nor IgG anti-EMA is as specific as IgA antibodies. Biopsy may still be clinically indicated if these tests are negative.

\section{For symptomatic cases}

If serology is negative but clinical suspicion persists (eg, chronic diarrhoea, faltering growth, IgA deficiency, positive family history), perform endoscopy and duodenal biopsies including biopsies of the duodenal cap (which may sometimes show diagnostic abnormalities where the more distal duodenum appears normal) to identify seronegative $\mathrm{CD}$ and other mucosal disorders.

\section{For asymptomatic cases}

In asymptomatic children with associated condition and negative serology, consider HLA typing.

If HLA DQ2/DQ8 positive: continue surveillance (optimum frequency for repeat blood testing unclear, but every 3 years is reasonable if asymptomatic) and perform endoscopy if symptomatic.

If HLA DQ2/DQ8 negative: development of CD highly unlikely. ${ }^{10}$ Discontinue regular antibody screening but clinical review if suggestive symptoms develop.

\section{Confirmation of the diagnosis}

Anti-tTG antibody positivity alone is insufficient for diagnosis. Therapeutic trials of GFD are NOT indicated if CD is suspected.

Children should not be started on a GFD on the basis of an antibody test alone, unless their clinical condition is so poor that treatment cannot safely be delayed (eg, coeliac crisis). It is recommended that such cases should be discussed with a paediatric gastroenterologist.

Confirmation of the diagnosis may differ between symptomatic and asymptomatic cases.

If symptomatic (summarised in figure 1):

First check IgA and IgA tTG.

A. If tTG negative and IgA normal, CD unlikely:

If IgA low, then further testing (eg, IgG tTG and possible biopsy) is required.

B. If tTG raised-but less than $10 \times$ upper limit of normal for assay:

Duodenal biopsy is required.

- At endoscopy, take four biopsies from D2 or lower and 1-2 from duodenal bulb (as patchy changes may be present). Ensure adequate gluten intake prior to testing with advice from dietician if necessary (see appendix 1).

C. If tTG raised-and greater than $10 \times$ upper limit of normal for assay:

Take further blood sample to check IgA-EMA and determine HLA-DQ2/HLA-DQ8 typing. If EMA+ and patient either DQ2 or DQ8, the diagnosis is confirmed without the need for a duodenal biopsy. If EMA antibody testing is not locally available, a second strongly positive tTG antibody may be substituted and serum saved for later EMA testing. 


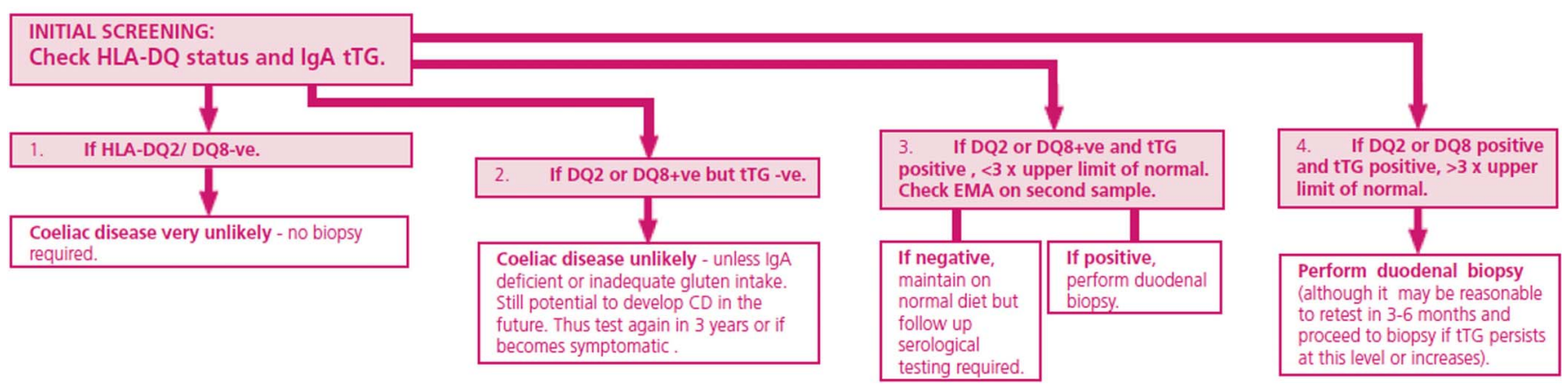

Figure 2 Outline stratagem for asymptomatic children with associated conditions.

Or: If asymptomatic but with associated conditions

(summarised in figure 2):

Explain implication of positive test and obtain document consent for testing. All such children will require a small intestinal biopsy for diagnosis to be confirmed. Three months challenge with adequate gluten intake (appendix 1) prior to testing is advised with option to expedite blood testing if patient develops symptoms.

First check HLA-DQ status and IgA tTG.

A. If HLA-DQ2/HLA-DQ8 negative:

CD very unlikely and no biopsy required

B. If DQ2 or DQ8 positive but $\mathrm{tTG}$ negative:

CD unlikely unless IgA deficient or inadequate gluten intake but still potential to develop $\mathrm{CD}$ in the future. Further testing required in 3 years or if becomes symptomatic.

C. If DQ2 or DQ8 positive and $\mathrm{tTG}$ positive, $<3 \times$ upper limit of normal:

Check EMA on second sample. If negative, maintain on normal diet but follow-up serological testing required. If positive, perform duodenal biopsy

D. If DQ2 or DQ8 positive and tTG positive, $>3 \times$ upper limit of normal:

Perform duodenal biopsy (although it may be reasonable to retest in 3-6 months and proceed to biopsy if tTG persists at this level or increases).

\section{Interpretation of small bowel histology}

The modified Marsh grading system is now accepted as the standard method of analysis. ${ }^{11}$

Villous atrophy with crypt hyperplasia and increased intraepithelial lymphocytes (IELs) >30/100 epithelial cells (Marsh type 3) characteristic of CD.

Increased IELs with crypt hyperplasia (Marsh type 2) compatible with CD:

- diagnosis strengthened by positive serology;

- if serology negative, reconsider CD after exclusion of other disorders.

Increased IELs with normal villous architecture (Marsh type 1) is non-specific for CD but

- diagnosis strengthened by strongly positive serology
If diagnosis uncertain

A. Negative serology and mild infiltrative changes, options are as follows:

- Perform HLA typing (DQ2 and DQ8) if not already checked.

- Consider repeat biopsy after further challenge with increased gluten intake (if very strong clinical suspicion but histology negative or non-classical, enteroscopy may be considered).

- Consider repeat serology and biopsy after trial of GFD.

B. Positive serology with normal biopsy requires:

- Follow-up and repeated (at least annual) assessment of tTG and micronutrient status. If tTG titre is high or increasing or micronutrient deficiency develops then repeat endoscopy is required. If high clinical suspicion of CD but biopsies still normal, consider need for referral for enteroscopy.

- Document basis for diagnosis in case notes (appendix 2).

- Document response to GFD in case notes at follow-up.

Who to treat with GFD

1. All symptomatic children with characteristic abnormal histology:

Benefits of GFD:

- Resolution of symptoms.

- Reversed bone demineralisation.

- Resolution of micronutrient deficiencies and likely better height gain.

- Decreased rate of delayed puberty, menstrual problems, subfertility, spontaneous abortions and low birth weight babies.

- Decreased rate of some intestinal cancers to normal population levels.

- Possible prevention of onset of other autoimmune conditions (evidence conflicting).

2. Asymptomatic children with a condition associated with CD and characteristic histology.

Benefits of GFD:

- Likely to reverse covert micronutrient deficiency and optimise bone mineralisation.

- Unclear whether diabetes control improves.

- No studies on long-term outcomes of GFD in children with associated conditions. 
COELIAC DISEASE CASE SUMMARY

File this sheet in the front of the case notes

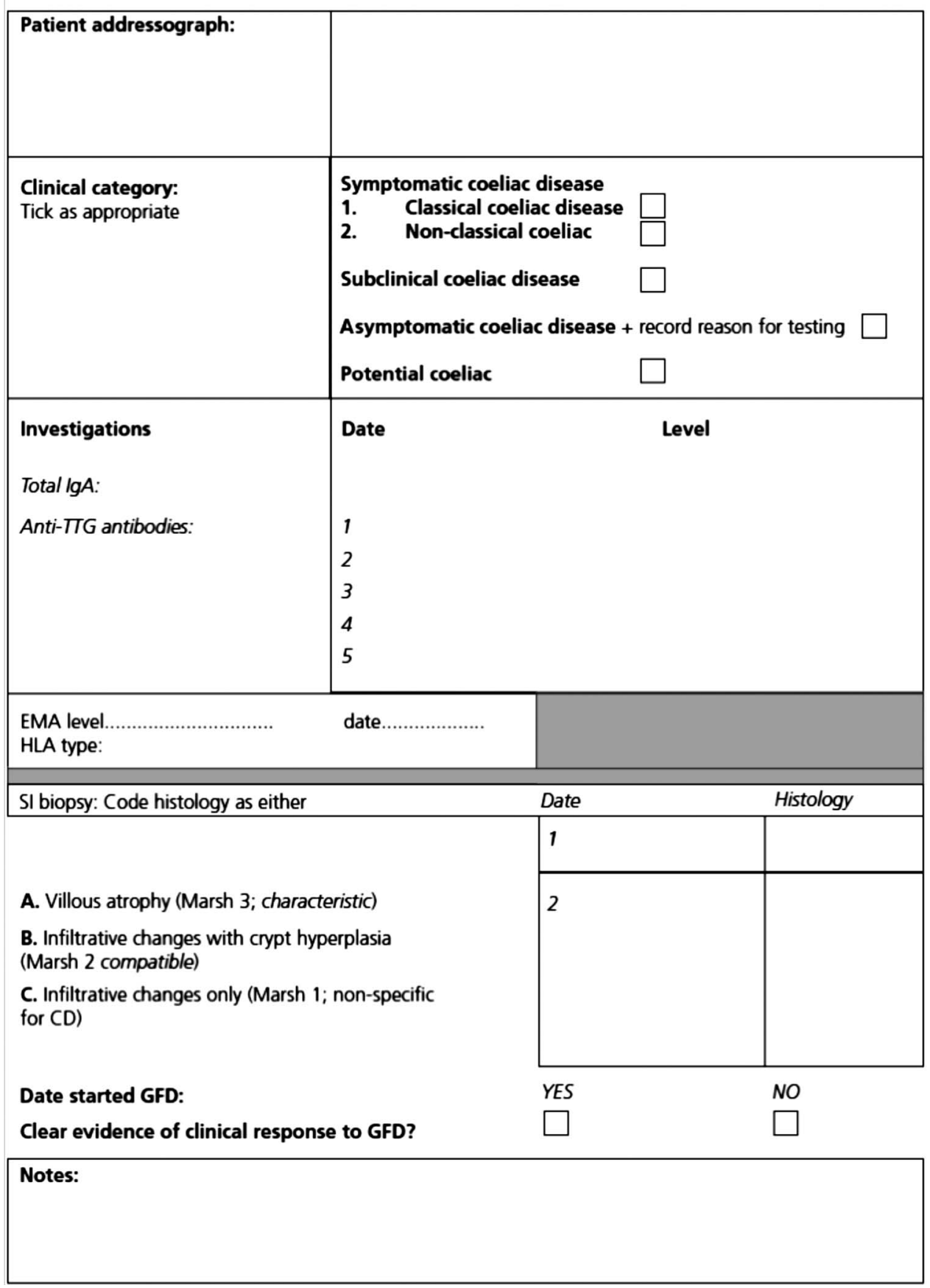

Figure 3 Suggested coeliac disease case summary for insertion in clinical notes.

How to treat

Start GFD after diagnosis confirmed by histology. We suggest that a case summary (figure 3 ) is inserted in the case notes. GFD will be required lifelong and needs regular paediatric dietetic support, ideally initiated within 1-2 weeks of diagnosis, followed up at 3-6 monthly intervals in the first year, and thereafter, annually. Additional input may be required if there are problems with dietary adherence. Compliance to GFD is likely to be improved by education of the child and family about the disease (we would strongly recommend that families join Coeliac UK) and by a proactive approach by paediatricians and dieticians in supporting a GFD and identifying children whose serology does not normalise within 12 months of commencing GFD.

- Sensitivity to gluten and acknowledgment of, symptoms after ingestion is variable between patients. Small amounts of gluten ingested regularly can cause mucosal changes even if patient feels asymptomatic.

From 2012, only foods that contain 20 ppm or less can be labelled as gluten-free. This may apply to both specialist substitute gluten-free products and processed foods that are naturally 
gluten-free products like soups, baked beans and crisps. The gluten-free label may also be used for uncontaminated oat products. Specialist substitute foods (eg, breads and pasta containing Codex wheat starch) containing between 21 and $100 \mathrm{ppm}$ gluten may be labelled as 'very low gluten'.

These guidelines are more stringent than previous UK definitions of gluten-free as $<200 \mathrm{ppm}$ and are covered by law.

- Oats are safe for most patients with CD, although around 5\% of patients will be sensitive to oats. It is essential that patients use only uncontaminated oats. These will be labelled gluten-free.

- Ideally establish patient on strict GFD excluding oats and consider reintroduction when baseline of wellness achieved, often after at least a year on GFD. Monitor carefully for signs or symptoms.

- Normalisation of tTG prior to commencing oats and continued low titres after reintroduction may provide further reassurance.

- Most coeliac patients tolerate Codex wheat starch and barley malt extract. Products containing barley malt extract must be below $20 \mathrm{ppm}$ to be labelled GF. Codex wheat starch can be used in both products labelled GF $<20$ or very low gluten 21-100. The $<20$ level should be safe for all unless they have a separate non-coeliac sensitivity to wheat. The 21-100 levels should be acceptable for most coeliacs, but not those with high sensitivity to gluten.

- Of note, Codex wheat starch is used in some gluten-free products available on prescription, so the suitability should be established prior to prescribing.

- Lactose-free diet is very rarely needed, although in some, temporary lactose intolerance can coexist and may need specific dietetic advice. More persistent lactose intolerance suggests the need for further assessment to exclude inadequate dietary compliance or additional pathology requiring separate treatment (eg, cow's milk sensitive enteropathy).

- Ensure easy access to paediatric dietitian(s)

Advise families to join Coeliac UK: http://www.coeliac.org.uk. Helpline: 0845305 2060. This charity offers various forms of support to families and its Food and Drinks Directory and monthly updates offers one of the easiest ways to obtain information about a wide range of suitable gluten-free manufactured foods.

\section{How to monitor}

The majority of patients/parents are well motivated and access good levels of support from Coeliac UK. Ongoing monitoring by an experienced paediatric dietitian and paediatric gastroenterologist or paediatrician with a special interest is still necessary to ensure long-term adherence.

- Postdiagnosis clinical assessment by a clinician and dietitian experienced in the care of children and young people with CD (symptoms, growth, physical examination, micronutrient status, adherence to GFD), dietary intake of calcium and iron and anti-tTG antibodies 6-12 months after starting GFD.

- Annual clinic assessment (as above), including anti-tTG antibodies annually (or biannually if stable long-term).

- Urgent clinical review if symptoms recur.

- Repeat anti-tTG antibodies may help to identify dietary non-adherence.

- Ensure transition to 'adult' team when appropriate.

\section{Gluten challenge to confirm diagnosis when initial diagnosis uncertain}

Routine rechallenge is not required if diagnosis is secure. If initial diagnosis is insecure and the child is on a gluten-free diet, then further testing is recommended.
HLA testing is a useful initial screen in these circumstances. If HLA-DQ2 and HLA-DQ8 negative, then the child is unlikely to have true CD. If HLA-DQ2 or HLA-DQ8 positive, then a formal gluten challenge will be required for clarification of the diagnosis.

Three months gluten challenge prior to testing is advised if asymptomatic with option to expedite blood testing when patient develops symptoms. A minimum duration of 4-6 weeks for those with symptoms during gluten challenge is recommended to maximise the likelihood of clear diagnosis. Biopsy should be performed when serology becomes positive or symptoms are difficult to tolerate. In the DQ- $2 / 8$ positive child who manifests clear symptoms and develops tTG greater than $10 \times$ the upper limit of normal, then it may be reasonable to retest a second sample for EMA in lieu of biopsy.

In the circumstances that an older child becomes uncertain about their diagnosis, then a gluten challenge may be indicated. However, a thorough review with the patient to inform them of the specific basis for their initial diagnosis is important prior to considering this.

If considering gluten challenge:

- Perform at age 6-7 years or when pubertal growth is complete.

- This is best managed by reintroducing a normal diet with adequate gluten content-See appendix 1 for recommended intake. Gluten powder (10-15 g/day) is an alternative, but is less palatable and difficult to obtain.

- Monitor symptoms and serum anti-tTG antibodies, repeating biopsy if serology becomes positive. Patients require follow-up for at least 2 years post challenge with serology at 6-month intervals if remaining symptom-free. Consider biopsy at 2 years even if asymptomatic. Patients should be advised that much later relapse may occur, ${ }^{12}$ and thus, they should be referred again for gastroenterological assessment if they ever develop symptoms suggestive of CD.

\section{Pneumococcal vaccine}

This is now recommended for patients with CD (Coeliac UK guidelines). Many children will already have had this as part of their routine immunisation programme.

\section{Author affiliations}

${ }^{1}$ Division of Metabolic and Vascular Health, Warwick Medical School, Coventry, UK

${ }^{2}$ Department of Child Health, University Hospital of Wales, Cardiff, UK

${ }^{3}$ Department of Paediatric Gastroenterology, Royal Liverpool Children's Hospital, Liverpool, UK

${ }^{4}$ Department of Paediatric Gastroenterology, Birmingham Children's Hospital, Brimingham, UK

${ }^{5}$ Department of Child Health, Royal Alexandra Hospital for Sick Children, Brighton, UK ${ }^{6}$ Department of Dietetics, University Hospital of North Staffordshire, Stoke, UK ${ }^{7}$ Department of Paediatric Gastroenterology, Royal Free Hospital, London, UK ${ }^{8}$ Department of Paediatric Gastroenterology, Royal Hospital for Sick Children, Edinburgh, UK

${ }^{9}$ Department of Paediatric Gastroenterology, Hospital for Sick Children, London, UK

${ }^{10}$ Department of Child Health, Epsom and St Helier NHS Trust, St Helier, UK

${ }^{11}$ Department of Child Health, North Tees and Hartlepool NHS Trust, North Tees, UK

${ }^{12}$ Department of Child Health, Norwich University Hospitals, Norfolk, UK

${ }^{13}$ Coeliac UK, High Wycombe, UK

${ }^{14}$ Department of Child Health, Royal Cornwall Hospital, Truro, UK

Acknowledgements The authors would like to thank Dr Mark Beattie, recent president of BSPGHAN, for his great support in the development of these guidelines.

Contributors The authors represent the Coeliac Disease Working Group of BSPGHAN. All authors contributed to the development of these guidelines through participation in the Working Group process. The manuscript was drafted by SM. SS is Chief Executive of Coeliac UK, who is promulgating these guidelines jointly with BSPGHAN.

Competing interests None.

Provenance and peer review Not commissioned; externally peer reviewed. 


\section{REFERENCES}

1 Hill ID, Dirks MH, Liptak GS, et al. Guideline for the diagnosis and treatment of celiac disease in children: recommendations of the North American Society for Pediatric Gastroenterology, Hepatology and Nutrition. JPGN 2005;40:1-19.

2 Ahmed M, Cosgrove M, Dale PJ, et al. Consensus report for the management of childhood coeliac disease in Wales. Welsh Paed J 2005;22:37-41.

3 Husby $S$, Koletzko $S$, Korponay-Szábó IR, et al. ESPGHAN guidelines for the diagnosis of coeliac disease in children and adolescents. An evidence-based approach. JPGN 2012;54:136-60.

4 Abadie V, Sollid LM, Barreiro LB, et al. Integration of genetic and immunological insights into a model of celiac disease pathogenesis. Annu Rev Immunol 2011;29:493-525.

5 Green PHR, Jabri B. Coeliac Disease. Lancet 2003;362:383-91.

6 Jabri B, Sollid LM. Mechanisms of disease: immunopathogenesis of celiac disease. Nat Clin Pract Gastroenterol Hepatol 2006;3:516-25.

7 Kaukinen K, Partanen J, Mäki M, et al. HLA-DQ typing in the diagnosis of celiac disease. Am J Gastroenterol 2002;97:695-99.

8 White LE, Bannerman E, McGrogan P, et al. Childhood coeliac disease diagnoses in Scotland 2009-2010: the SPSU project. Arch Dis Child 2013;98:52-6.

9 Jenkins HR, Murch SH, Beattie RM, et al. Diagnosing coeliac disease. Arch Dis Child 2012;97:393-4.

10 Bonamico M, Ferri M, Mariana $\mathrm{P}$, et al. Serologic and genetic markers of celiac disease: a sequential study in the screening of first degree relatives. JPGN 2006:42:150-4.

11 Oberhuber G, Granditsch G, Vogelsgang H. The histopathology of coeliac disease: time for a standardized report scheme for pathologists. Eur J Gastroenterol Hepatol 1999;11:1185-94.

12 Matysiak-Budnik T, Malamut G, de Serre NP, et al. Long-term follow-up of 61 coeliac patients diagnosed in childhood: evolution toward latency is possible on a normal diet. Gut 2007;56:1379-86.

13 Ludvigsson JF, Leffler DA, Bai JC, et al. The Oslo definitions for coeliac disease and related terms. Gut 2013;62:43-52.

\section{APPENDIX 1}

Ensuring adequate gluten intake for initial biopsy (or during gluten challenge in older children). Parents should be advised that this may exacerbate symptoms temporarily but increases the likelihood of a secure diagnosis. The input of a paediatric dietician should be sought if there is any doubt whether the child is taking sufficient gluten for a secure diagnosis during challenge.

\section{Recommended for most children}

Typically, $10-15 \mathrm{~g}$ gluten per day is required for adequate intake in most children (eg, 2-3 g gluten are contained in one medium bread slice, one Weetabix or Shredded Wheat, two rusks or digestives and four tablespoons of cooked pasta). General advice would be to provide at least two (preferably three) gluten-containing meals per day, containing at least $5 \mathrm{~g}$ gluten per meal.

2. In infants and young children

It may be impossible to achieve intakes of $10 \mathrm{~g}$ gluten daily in young children. Thus advice should be to provide two gluten containing meals per day, where bread, pasta, gluten containing breakfast cereal form the carbohydrate component of the meal, for example, half a Weetabix for breakfast and one slice of bread for lunch should be sufficient.

Note: using inadvertent gluten-containing foods alone, for example, gravy on a meal is insufficient.

\section{APPENDIX 2 \\ ACCEPTED NOMENCLATURE FOR COELIAC DISEASE (OSLO CLASSIFICATION). ${ }^{13}$}

Classic coeliac disease presents with signs and symptoms of malabsorption. Diarrhoea, steatorrhoea, weight loss, iron deficiency anaemia or growth failure is required.

Non-classic coeliac disease presents without signs or symptoms of malabsorption (eg, abdominal pain, constipation, transaminitis etc).

Symptomatic coeliac disease is characterised by clinically evident gastrointestinal or extraintestinal symptoms.

Asymptomatic coeliac disease is found in persons with positive diagnostic tests for coeliac disease but with no intestinal or systemic symptoms and no overt clinical response to gluten-free diet.

Subclinical coeliac disease occurs without signs or symptoms sufficient to have triggered screening in routine clinical practice. However, previously unrecognised symptoms such as fatigue may improve on a gluten-free diet.

Potential coeliac disease is found in children with positive coeliac serology but with normal small intestinal biopsies (it is important to ensure that adequate biopsies have been taken, including the duodenal bulb). Such persons are at increased risk of progressing to coeliac disease and require long-term follow-up.

Coeliac disease autoimmunity is defined by positive tTG or EMA serology on two occasions without definitive diagnosis. Such children will require small bowel biopsy to determine whether they have coeliac disease or potential coeliac disease.

Refractory coeliac disease is characterised by persistence or recurrence of malabsorptive symptoms and signs together with villous atrophy on small intestinal biopsy despite maintenance of an adequate gluten-free diet for over 12 months.

Non-coeliac gluten sensitivity occurs in persons with a reproducibly adverse response to ingesting gluten, either symptomatic or on small intestinal biopsy, in whom coeliac disease has been excluded. Potential causes include non-IgE-mediated food allergy.

Terms such as silent coeliac disease, latent coeliac disease and overt coeliac disease are no longer recommended. 